\title{
Research on the Teaching Method of Process Synchronization of Computer Operating Systems
}

\author{
Jiuru WANG, Lihua HAN, Xiaojie WANG and Yulong ZUO \\ School of Information, Linyi University, Linyi Shandong, China 276000
}

Keywords: Operating Systems, Process synchronization, E-R diagram, Teaching research.

\begin{abstract}
In the teaching process of the course of operating systems, process synchronization is both a key and the biggest difficulty. This paper, through transforming the definition of E-R diagram in database, proposes process synchronization teaching method for computer operating systems based on modified E-R diagram. The method formulates process entities in rectangles, among which a diamond indicates links between entities and an oval represents the semaphore that generates association; thus able to clear reveal the connection between process entities as well as that within the entity. Teaching practice shows that this method is not only advantageous to the description and analysis of the process synchronization problem, but also helpful for students to master the ideas of process synchronization design. In addition, it can improve their ability to solve the problems related to process synchronization.
\end{abstract}

\section{Introduction}

Computer Operating Systems is one of the core and foundation courses for colleges and junior colleges students majoring in computer science. Improving the teaching quality of this course is very important to improve students' computer literacy and to cultivate qualified computer professional talents. In the process of teaching this course, process synchronization is both a key and the biggest difficulty. The causes lie in that: (1) preceding courses rarely involves in related design thoughts; (2) process synchronization concept is abstract; and (3) problems related to process synchronization are characteristic of a variety of forms and complex content. In expressing of a subject, process synchronization often relates it to real life, the description of which is a little complicated. As no unified methodology has been arrived in the process of teaching the subject, teachers all depends on their individual understanding of it. Thus, it is easy to bring about such a situation that students seem to know but they not really know how to start.

Based on the above causes and combined with the author's teaching experience of many years, this paper puts forward process synchronization teaching method for the course of Computer Operating Systems based on modified E-R diagram. First of all, it transforms the "Entity-Relation" diagram commonly used in database design and works out the E-R diagram suitable to process synchronization analysis. Second, it analyzes the producer-consumer problem with the help of the modified E-R diagram. Through teaching practice it has been proved that with the help of the modified E-R diagram synchronization process model can be intuitively and visually described and the analysis of process synchronization problems can be simplified so that it is helpful to understand and master the essence of process synchronization. 


\section{Modifying E-R Diagram}

E-R diagram is the short form of Entity-Relationship Diagram, which is an effective method to describe the model on the concept structure of the real world. The basic elements that constitute the diagram include entity, contact and attribute. For clearly manifesting the relationship between the process entities in process synchronization, this paper makes modification for the E-R diagram as below:

Entity: refers to a process of different types that participates in process synchronization. Its name is used to abstract and describe the similar process entities and inside the rectangular box set forth the name of the entity as shows in Fig. 1.

\section{Name of a \\ Process Entity}

Figure 1 Process Entity Legend

Relationship: also called relation or connection, reflects the relationship inside the process and external relationship between processes. The former refer to relationship between both the similar process entities while that between processes is the relationship between different process entities. In the modified E-R diagram, a diamond is used to indicate relationship, inside which the type of relationship is marked out (as synchronous/mutually exclusive or mutex relationship). The diamonds are connected to process entities with arrowed or arrowless lines respectively, as shows in Fig. 2.

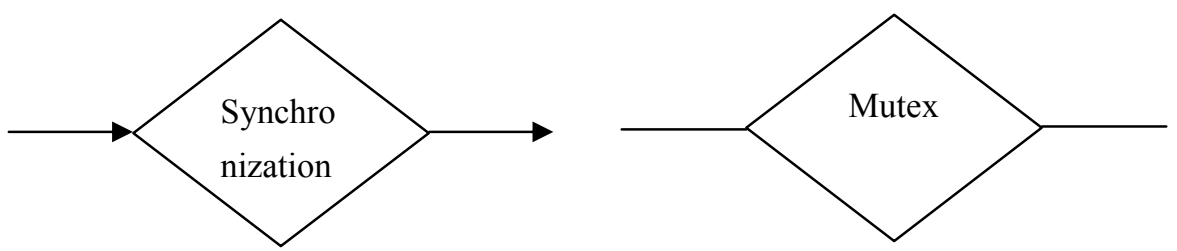

Figure 2 Entity Relationship Legend

Attribute: described as the name of the semaphore brought by the occurrence of relationship between process entities, indicated by an ellipsis which is connected to corresponding relationship with non-arrowed lines, shown in Fig. 3.

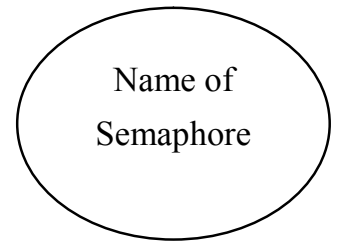

Figure 3 Relationship Attribute Legend

\section{Drawing Steps}

In order to get a clear description in the multiprogramming environment and of multiple processes in the same system that share the system resources or their mutual cooperative relationship for accomplishing a task, this paper put forwards a problem-solving idea of "abstracting entities and clarifying relationship".

Types of Abstracting Entitles. According to the description of the problem of process synchronization, this step analyzes the types of different process entities that unsolved practical problems involve and abstracts them in representation of rectangles respectively. 
Clarifying Relationship between Process Entities. According to the description of activities of various process entities, this step judges the relationship between process entities and uses diamonds to indicate them. The relationships are ranged into:

(1) Mutual exclusion: Multiple programs in concurrent execution when sharing system resources, such as CPU, I/O and other devices, lead to mutual check between themselves. In order to ensure that these processes can run in an orderly way, this kind of resources in the system must be allocated unfriendly by the system, in other words, the user should lodge a request before use and no direct use is allowed.

(2) Synchronization: Some executive programs establish two or more processes for accomplishing a task, which will cooperate to accomplish the same task. Direct restriction relationship between the processes derives the mutual cooperation between them.

(3) Coexistence of mutual exclusion and synchronization.

Different Method for Different Relationships. 'There are three different methods for solving problems of different relationship as below:

a. Solving Steps to Mutual Exclusion Problems

(1) Clarify critical resources for competition of process entities in mutual exclusion and the critical region to access them;

(2) Use attribute to indicate the semaphore of mutual exclusion (e.g., mutex) and setting initial value of the attribute as $\mathrm{n}$ ( $\mathrm{n}$ is the number of critical resources owned by the system);

(3) Set wait (mutex) primitive as the entry area before access to the critical region while set signal (mutex) primitive as the exit area after access to the critical region;

(4) wait (mutex) and signal (mutex) are required to occur in pairs in a same process, which indicate utilization and release of critical resources respectively.

b. Solving Steps to Synchronization Problems

(1) Determine the subsequence between process entities;

(2) Use attribute to indicate the semaphore of synchronization (e.g., empty) and set the initial value of the attribute as $\mathrm{n}$ or 0 ( $\mathrm{n}$ is an integer to indicate the initial value of resources that can be applied for);

(3) Insert wait (empty) primitive before the arrowhead as the entry area while insert signal (empty) primitive after string notch as the exit area;

(4) Normally, for process entities waiting for the signal, the initial value of wait () primitive semaphore is 0 ; otherwise it is $\mathrm{n}$. It should be noted that wait (empty) and signal (empty) occur in pairs in different processes.

c. Solving Steps to Problems of Coexistence of Mutual Exclusion and Synchronization

(1) If the relationships of mutual exclusion and synchronization coexist between process entities, then draw reference from solving steps to mutual exclusion and synchronization problems;

(2) Pay attention to the following four points: (1) if two wait () primitives exist in the entry area of entities of the same process, execute synchronous relationship first and then mutex relationship. Otherwise, there might be a deadlock ; (2) if there are two signal () primitives in the exit area of entities of the same process, although no strict execution sequence, the standard execution order is as follows: executing the mutex relationship first and the synchronization relationship second. Otherwise it will affect the system performance; (3) wait () and signal () primitives may be distributed in entities of different processes, but they must come in pairs or a deadlock may appears. 


\section{An Analysis of the Producer-Consumer Problem}

What the Producer-Consumer problem describes is like the following. A group of producer processes continuously provide products for a group of consumer processes through a buffer pool with $\mathrm{n}$ buffer cycles, during which consumer processes do not need to get access to an empty buffer to get products, nor producer processes need to launch products in buffer areas filled with products.

Solutions are given as below:

(1) From the above description, it can be seen that the system comprise two kinds of process entities, namely, one of the producer and the other of the consumer. Therefore, they are indicated by two rectangles respectively in the E-R diagram.

(2) Because there are two kinds of sequence relationship between the producer and the consumer, namely, from the producers to the consumer process and from the producer to the consumer process respectively, they are of synchronization relationship that includes two kinds.

(3) In terms of the two kinds of relationship, set the attribute full (initial value as 0 ) as semaphore for the former and empty (initial value as number of buffers $n$ ) as semaphore.

(4) The E-R diagram of the Producer-Consumer process is hence elicited as shown in Fig. 4.

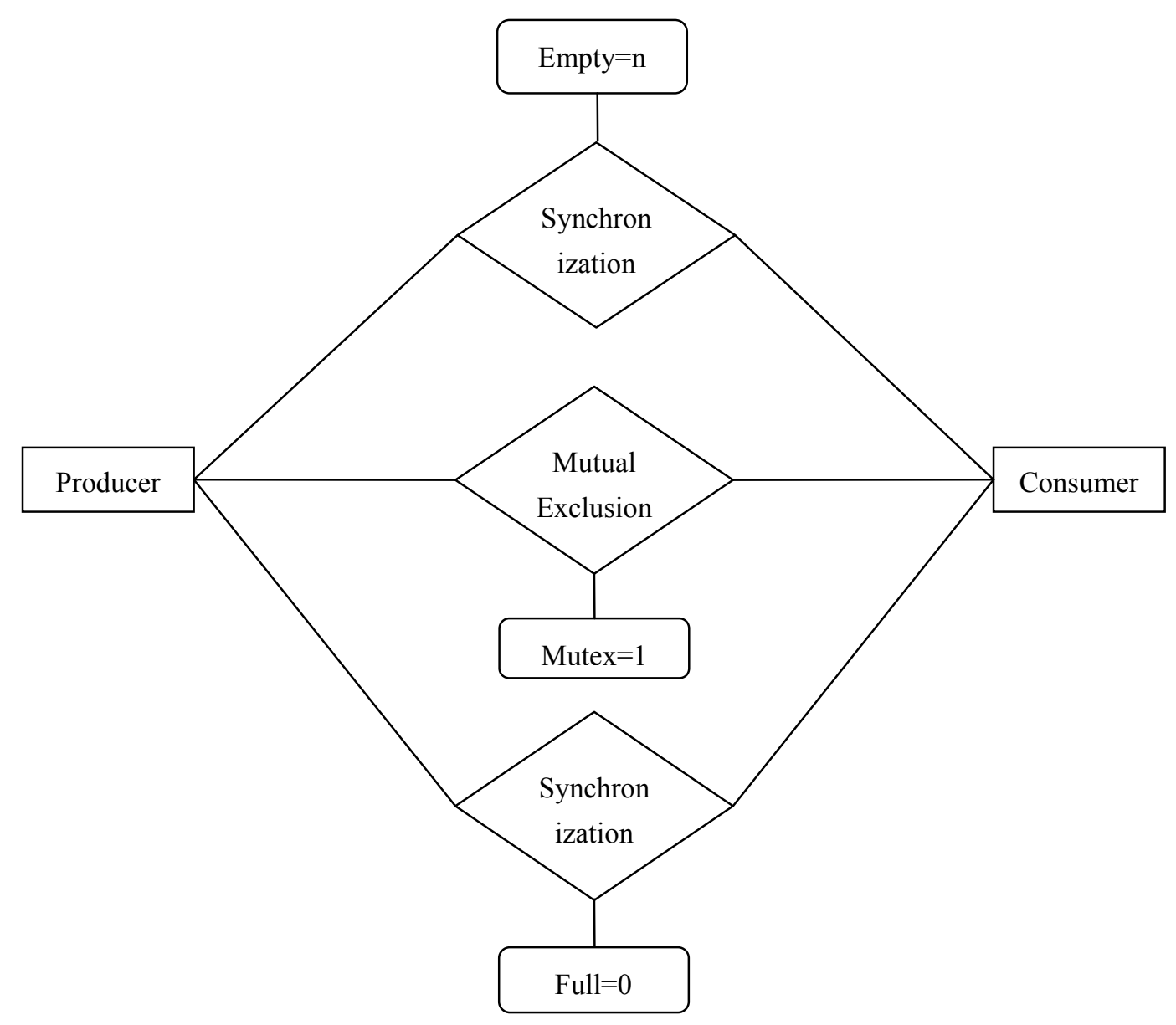

Figure 4 E-R Diagram of the Producer-Consumer Process 
(5) Reference codes are given as follows:

\begin{tabular}{|c|c|}
\hline void proceducer( ) \{ & void consumer( ) \{ \\
\hline do \{ & do \{ \\
\hline producer an item nextp; & wait(full); \\
\hline ...... & wait(mutex); \\
\hline wait(empty); & nextc $=$ buffer[out $]$ \\
\hline wait(mutex); & out $=($ out +1$) \bmod n$ \\
\hline buffer $[\mathrm{in}]=$ nextp; & signal(mutex); \\
\hline in $=($ in +1$) \bmod n$ & signal(empty); \\
\hline signal(mutex); & consumer the item in nextc; \\
\hline signal(full); & ...... \\
\hline \} while (TRUE); & \} while (TRUE); \\
\hline \} & \} \\
\hline
\end{tabular}

\section{Summary}

Through the design of reformed E-R diagram this paper puts forward a synchronization analysis method in terms of transformation of the computer operating system processes. This method is easy to learn and the legends can clearly represent the connection between process entities as well as within the entities. Teaching practice shows that using modified E-R diagram to analyze process synchronization problems is not only beneficial to the description and analysis of such problems but also helpful for students to master design ideas of process synchronization and to improve their ability to solve such problems.

\section{Acknowledgement}

This research is sponsored by Shandong Provincial Natural Science Foundation, China (Project No.: ZR2014FL012), Shandong Provincial Key Laboratory Open Foundation of Intelligent Computing Technology of Network Environment and Initial Research Fund for PhD Programs of Linyi University (Project No.: LYDX2014BS006).

\section{About the first author}

Jiuru WANG, male, $\mathrm{PhD}$, lecturer, whose research direction is network information security, sensor network and internet of things. 


\section{References}

[1] X. D. Tang, H. B. Liang, F. P. Zhe and Z. Y. Tang, Computer Operating Systems, fourth ed., Xidian University Publishing House, Xi'an, 2014.

[2] Y. H. Zhang, X. Li and H. L. Ma, The teaching reform of the course design of operating system in the Android platform, Computer Education. 8 (2014) 32-35.

[3] X. P. Liu, X. Chen, Q. Lu and W. D. Tian, A probe into the teaching method of "inspiration - inquiry" for the course of operating system, Computer Education. 2 (2011) 50-53.

[4] J. F. Qiu, J. Xie, L. S. Li, J. W. Wang and W. Li, Discussion on teaching of the "reader to reader" problem in the course of operating system, Computer Education. 22 (2011) 56-58.

[5] Z. M. Li, Y. D. Zhao and H. Di, Teaching Practice of Operating System Process Synchronization in Advances in Computer Research-Annual Conference Proceedings of Henan Computer Federation 2010, Electronic Industry Press, Beijing and Henan Computer Federation, Anyang, 2010, p. 5.

[6] J. M. Ji, S. P. Li and H. Qian, A Brief analysis of the examination paper of operating system of the national unified entrance examination of graduate students in 2009, Computer Education. 14 (2009) 44-46.

[7] H. D. Jin, Y. L. Xu. Research on the teaching of process mutex and synchronization in the course of "computer system", Computer Education. 14 (2009) 60-62. 